\title{
Distributed Stochastic Nonconvex Optimization and Learning based on Successive Convex Approximation
}

\author{
Paolo Di Lorenzo and Simone Scardapane* \\ March 4, 2022
}

Note: This paper was presented at the 2019 Asilomar Conference on Signals, Systems, and Computers. Due to a miscommunication, the paper was mistakenly omitted from the original submission to IEEE Xplore. It is now included as part of the conference record.

\begin{abstract}
We study distributed stochastic nonconvex optimization in multi-agent networks. We introduce a novel algorithmic framework for the distributed minimization of the sum of the expected value of a smooth (possibly nonconvex) function-the agents' sum-utilityplus a convex (possibly nonsmooth) regularizer. The proposed method hinges on successive convex approximation (SCA) techniques, leveraging dynamic consensus as a mechanism to track the average gradient among the agents, and recursive averaging to recover the expected gradient of the sum-utility function. Almost sure convergence to (stationary) solutions of the nonconvex problem is established. Finally, the method is applied to distributed stochastic training of neural networks. Numerical results confirm the theoretical claims, and illustrate the advantages of the proposed method with respect to other methods available in the literature.
\end{abstract}

\section{Introduction}

Recent years have witnessed a surge of interest in distributed optimization methods for multiagent systems. In the stochastic setting, many such problems can be formulated as the cooperative minimization of the expected agents' sum-utility $F$ plus a regularizer $G$ :

$$
\begin{aligned}
& \underset{\mathbf{x}}{\operatorname{minimize}} \mathbb{E}[F(\mathbf{x}, \xi)]+G(\mathbf{x}) \\
& \text { subject to } \mathbf{x} \in \mathcal{K},
\end{aligned}
$$

*Authors are with the Department of Information Engineering, Electronics, and Telecommunications, Sapienza University of Rome, Via Eudossiana 18, 00184, Rome, Italy. E-mail: \{paolo.dilorenzo,simone.scardapane\}@uniroma1.it. This work was funded by Sapienza project n. RP11816431B9E1BC. 
where $F(\mathbf{x}, \xi) \triangleq \sum_{i=1}^{I} f_{i}(\mathbf{x}, \boldsymbol{\xi})$ is the sum-utility function, with each $f_{i}(\mathbf{x}, \boldsymbol{\xi})$ being the smooth (possibly nonconvex, nonseparable) cost function of agent $i \in\{1, \ldots, I\}$ that depends on the variable $\mathbf{x} \in \mathbb{R}^{p}$ and a random vector $\xi$, whose probability distribution is defined on $\mathcal{D} \subseteq \mathbb{R}^{p}$; $G$ is a convex (possibly nonsmooth, nonseparable) function; and $\mathcal{K} \subseteq \mathbb{R}^{p}$ is closed and convex. Usually the nonsmooth term is used to promote some extra structure in the solution, typically sparsity.

Network-structured optimization problems in the form (1) are found widely in several engineering areas, including sensor networks information processing, communication networks, multi-agent control and coordination, and distributed machine learning, just to name a few. Common to these problems is the necessity of performing a decentralized computation/optimization, due to the large size of the network and volume of data, energy constraints, and/or privacy issues. Motivated by these observations, this paper aims to develop a provable solution method for the general class of nonconvex stochastic problems (1), in the following distributed setting: i) the network of agents is modeled as a directed (strongly connected) graph; ii) agents know their local functions $f_{i}$ only, the common regularizer $G$, and the feasible set $\mathcal{K}$; and iii) only communications between single-hop neighbors are possible. Related works. Distributed solution methods for convex and deterministic instances of Problem (1) have been widely studied in the literature; they are usually either primal (sub)gradientbased methods [1-3], or primal-dual schemes, e.g., [4]. Similarly, distributed strategies for convex and stochastic instances of (1) are either diffusion adaptation schemes [5-8], or ADMM algorithms $[9,10]$. The literature on distributed nonconvex optimization is much more recent. The nonconvex and deterministic setting includes: i) primal gradient-based methods [11]; ii) Frank-Wolfe algorithms [12]; iii) SCA methods [13]; proximal primal-dual algorithms [14]; and distributed annealing schemes [15]. Finally, nonconvex and stochastic instances of (1) have been considered only very recently in [16-20]. In particular, the works in $[16,17]$ illustrate how distributed stochastic gradient algorithms achieves agreement at linear rate while escaping saddle points. The work in [19] provide sufficient conditions to guarantee asymptotic mean-square convergence of distributed stochastic gradient methods, considering twice differentiable objective functions. Finally, the work in [20] propose a firstorder distributed algorithm based on gradient-tracking, which finds stationary points with guaranteed convergence rate.

Contributions. All previous art on distributed stochastic nonconvex optimization is based on first-order methods that exploit only gradient information of the objective functions $f_{i}$, and does not consider constraints. This paper introduces the first distributed (best-responsebased) algorithmic framework for the distributed, stochastic, nonconvex, constrained optimization in the general form (1). The crux of the framework is a convexification-decomposition technique that hinges on SCA methods [13,21], while leveraging dynamic consensus as a gradient tracking mechanism, and recursive average to asymptotically recover the gradient of the expected loss function; we will term it as Stochastic in-Network succEssive conveX approximaTion algorithm (S-NEXT). Almost sure convergence to (stationary) solutions of the nonconvex problem (1) is established. Numerical simulations on distributed stochastic training of neural network models confirm the theoretical results, and assess the performance of the proposed method over real datasets. 


\section{In-Network Stochastic Nonconvex Optimization via SCA}

Consider a network composed of $I$ autonomous agents aiming to cooperatively and distributively solve Problem (1).

Assumption A. We make the following blanket assumptions:

(A1) The set $\mathcal{K}$ is (nonempty) closed and convex;

(A2) Each $f_{i}$ is $C^{1}$ (possibly nonconvex) on $\mathcal{K}$;

(A3) $\nabla f_{i}$ is Lipschitz continuous and bounded on $\mathcal{K}$;

(A4) $G$ is a convex function (possibly nondifferentiable) with bounded subgradient on $\mathcal{K}$;

(A5) $U$ is coercive;

(A6) $\xi$ is a bounded i.i.d. random vector defined on set $\mathcal{D}$.

Assumptions above are standard and satisfied by many practical problems. Note that $f_{i}$ 's need not be convex. In the following, we also make the blanket assumption that each agent $i$ knows only its own $f_{i}$ (but not $F$ ), the common $G$, and the feasible set $\mathcal{K}$.

On network topology: The network of the agents is modeled as a directed graph $\mathcal{G}=$ $(\mathcal{V}, \mathcal{E})$, where $\mathcal{V}=\{1, \ldots, I\}$ is the vertex (i.e., agent) set, and $\mathcal{E}$ is the set of edges. The neighborhood of agent $i$ (including node $i$ ) is defined as $\mathcal{N}_{i}=\{j \mid(j, i) \in \mathcal{E}\} \cup\{i\}$; it sets the communication pattern between single-hop neighbors: agents $j \neq i$ in $\mathcal{N}_{i}$ can communicate with node $i$. We introduce the weights $w_{i j}$ matching the graph $\mathcal{G}$, i.e. $w_{i j}>0$ if $j \in \mathcal{N}_{i}$.

We also define the matrix $\mathbf{W} \triangleq\left(w_{i j}\right)_{i, j=1}^{I}$. We make the following weak assumptions on the network connectivity.

(A7) The graph $\mathcal{G}$ is connected. Furthermore, the weight matrix $\mathbf{W}$ satisfies $\mathbf{W} \mathbf{1}=\mathbf{1}$ and $\mathbf{1}^{T} \mathbf{W}=\mathbf{1}^{T}$.

Our goal is to develop an algorithm that converges to stationary solutions of Problem (1) while being implementable in the above distributed setting. To shed light on the core idea of our decomposition technique, we introduce first an informal and constructive description of the proposed scheme.

\subsection{Development of S-NEXT: A constructive approach}

Devising distributed solution methods for Problem (1) faces three main challenges, namely: the impossibility to evaluate the expectation accurately (e.g., because the statistics of the random variables are unknown and/or the computational complexity is prohibitive), the nonconvexity of $F$, and the lack of global information on $F$. To cope with these issues, we propose to combine SCA techniques (Step 1 below), recursive averaging (Step 2), and dynamic consensus mechanisms (Steps 3 and 4), as described next. 
Step 1 (local SCA optimization): Each agent $i$ maintains a local estimate $\mathbf{x}_{i}$ of the optimization vector $\mathbf{x}$ that is iteratively updated. Solving directly Problem (1) may be too costly (due to the expectation and the nonconvexity of $F$ ) and is not even doable in a distributed setting (because of the lack of knowledge of the whole $F$ ). One may then prefer to approximate Problem (1), in some suitable sense, in order to permit each agent to compute locally and efficiently the new iteration. Thus, to handle the nonconvexity of $F$ at every iteration $t$, given the local estimate $\mathbf{x}_{i}^{t}$, each agent $i$ should solve the following strongly convex optimization problem:

$$
\widehat{\mathbf{x}}_{i}^{t} \triangleq \underset{\mathbf{x}_{i} \in \mathcal{K}}{\operatorname{argmin}} \mathbb{E}\left[\widetilde{F}_{i}\left(\mathbf{x}_{i} ; \mathbf{x}_{i}^{t}, \boldsymbol{\xi}\right)\right]+G\left(\mathbf{x}_{i}\right)
$$

where $\widetilde{F}_{i}\left(\mathbf{x}_{i} ; \mathbf{x}_{i}^{t}, \boldsymbol{\xi}\right)$ is a suitably chosen strongly convex surrogate of the nonconvex original $F(\mathbf{x}, \xi)$, which may depend on the current iterate $\mathbf{x}_{i}^{t}$. The main idea behind (2) is to compute stationary solutions of Problem (1) as fixed-points of the mappings $\widehat{\mathbf{x}}_{i}(\bullet)$. The next proposition addresses the question about the connection between such fixed-points and stationary solution; its proof follows the same steps as [22, Prop. 8(b)] and thus is omitted.

Proposition 1 Given Problem (1) under A1-A6, suppose that $\widetilde{F}_{i}$ satisfies the following conditions:

(F1) $\widetilde{F}_{i}(\bullet ; \mathbf{y}, \xi)$ is uniformly strongly convex on $\mathcal{K}$;

(F2) $\nabla \widetilde{F}_{i}(\mathbf{x} ; \mathbf{x},, \xi)=\nabla F(\mathbf{x}, \xi)$ for all $\mathbf{x} \in \mathcal{K}, \xi \in \mathcal{D}$;

(F3) $\nabla \widetilde{F}_{i}(\mathbf{x} ; \bullet, \xi)$ is uniformly Lipschitz continuous on $\mathcal{K}$.

Then, the set of fixed-point of $\widehat{\mathbf{x}}_{i}(\bullet)$ coincides with that of the stationary solutions of $(1)$. Therefore, $\widehat{\mathbf{x}}_{i}(\bullet)$ has a fixed-point.

Conditions F1-F3 are quite natural: $\widetilde{F}_{i}$ should be regarded as a (simple) convex, approximation of $F$ at the point $\mathbf{x}$ that preserves the first order properties of $F$.

Step 2 (Recursive Averaging): The issue with (2) is that usually the expectation cannot be computed in closed form. To deal with it, we follow the approach proposed in [23]. Thus, given the realization of the random variable $\xi$ at time $t$, i.e., $\xi^{t} \in \mathcal{D}$, we propose to build the sample approximation of $\mathbb{E}\left[\widetilde{F}_{i}\left(\mathbf{x}_{i} ; \mathbf{x}_{i}^{t}, \boldsymbol{\xi}\right)\right]$ as:

$$
\bar{F}_{i}\left(\mathbf{x}_{i} ; \mathbf{x}_{i}^{t}, \xi^{t}\right)=\rho^{t} \widetilde{F}_{i}\left(\mathbf{x}_{i} ; \mathbf{x}_{i}^{t}, \xi^{t}\right)+\left(1-\rho^{t}\right) \mathbf{d}_{i}^{t^{T}}\left(\mathbf{x}_{i}-\mathbf{x}_{i}^{t}\right)
$$

where $\rho^{t}$ is a suitably chosen step-size sequence, and $\mathbf{d}_{i}^{t}$ is an online estimate of the gradient of $\mathbb{E}\left[F\left(\mathbf{x}_{i}, \xi\right)\right]$ that is recursively updated as:

$$
\mathbf{d}_{i}^{t+1}=\left(1-\rho^{t}\right) \mathbf{d}_{i}^{t}+\rho^{t} \nabla F\left(\mathbf{x}_{i}^{t}, \xi^{t}\right) .
$$

Then, using (3) in (2), each node $i$ at time $t$ solves the following strongly convex optimization problem:

$$
\widehat{\mathbf{x}}_{i}^{t} \triangleq \underset{\mathbf{x}_{i} \in \mathcal{K}}{\operatorname{argmin}}\left\{\rho^{t} \widetilde{F}_{i}\left(\mathbf{x}_{i} ; \mathbf{x}_{i}^{t}, \xi^{t}\right)+\left(1-\rho^{t}\right) \mathbf{d}_{i}^{t T}\left(\mathbf{x}_{i}-\mathbf{x}_{i}^{t}\right)+G\left(\mathbf{x}_{i}\right)\right\}
$$


where $\mathbf{d}_{i}^{t}$ is updated as in (4).

Step 3 (Gradient Tracking): The SCA step in (5) handles the expectation and the nonconvexity in (1). Nevertheless, (5) still cannot be computed locally by node $i$ because of the lack of global information needed to build $\widetilde{F}_{i}\left(\mathbf{x}_{i} ; \mathbf{x}_{i}^{t}, \xi^{t}\right)$, and to update $\mathbf{d}_{i}^{t}$ in (4). To cope with the first issue (i.e., the choice of $\widetilde{F}_{i}$ ), since node $i$ has knowledge only of $f_{i}$, writing $F\left(\mathbf{x}_{i}, \xi\right)=f_{i}\left(\mathbf{x}_{i}, \xi\right)+\sum_{j \neq i} f_{j}\left(\mathbf{x}_{i}, \xi\right)$, leads naturally to a $\widetilde{F}_{i}$ wherein the (possibly) nonconvex $f_{i}\left(\mathbf{x}_{i}, \xi\right)$ is replaced by a convex surrogate $\widetilde{f}_{i}\left(\mathbf{x}_{i} ; \mathbf{x}_{i}^{t}, \xi\right)$ and $\sum_{j \neq i} f_{j}\left(\mathbf{x}_{i}, \xi\right)$ is linearized around the current iterate $\mathbf{x}_{i}^{t}$. More formally, each agent $i$ solves the subproblem: given $\mathbf{x}_{i}^{t}$,

$$
\widehat{\mathbf{x}}_{i}^{t} \triangleq \underset{\mathbf{x}_{i} \in \mathcal{K}}{\operatorname{argmin}}\left\{\rho^{t}(\underbrace{\widetilde{f}_{i}\left(\mathbf{x}_{i} ; \mathbf{x}_{i}^{t}, \xi^{t}\right)+\pi_{i}\left(\mathbf{x}_{i}^{t}, \xi^{t}\right)^{T}\left(\mathbf{x}_{i}-\mathbf{x}_{i}^{t}\right)}_{\widetilde{F}_{i}\left(\mathbf{x}_{i} ; \mathbf{x}_{i}^{t}, \xi^{t}\right)})+\left(1-\rho^{t}\right) \mathbf{d}_{i}^{t T}\left(\mathbf{x}_{i}-\mathbf{x}_{i}^{t}\right)+G\left(\mathbf{x}_{i}\right)\right\},
$$

where

$$
\pi_{i}\left(\mathbf{x}_{i}^{t}, \xi^{t}\right) \triangleq \sum_{j \neq i} \nabla_{\mathbf{x}} f_{j}\left(\mathbf{x}_{i}^{t}, \xi^{t}\right) .
$$

It is easy to check that $\widetilde{F}_{i}$ in (6) satisfies F1-F3 if also $\widetilde{f}_{i}$ satisfies them. An appropriate choice of $\widetilde{f}_{i}$ depends on the problem at hand and on computational requirement. The computation of $\widehat{\mathbf{x}}_{i}^{t}$ in (6) is still not fully distributed, because the evaluation of $\pi_{i}\left(\mathbf{x}_{i}^{t}, \xi^{t}\right)$ in (7) and the update of $\mathbf{d}_{i}^{t}$ in (4) would require the knowledge of all $\nabla f_{j}\left(\mathbf{x}_{i}^{t}\right)$, which is not available locally at node $i$. This lack of global knowledge can be solved exploiting dynamic average consensus methods [24], which enable to track the network average gradient via local exchange of information between neighbors, as proposed in [13]. In particular, letting $\mathbf{y}_{i}^{t}$ be the local estimate at agent $i$ for $\overline{\nabla f}\left(\mathbf{x}_{i}^{t}, \xi^{t}\right)=(1 / I) \sum_{j=1}^{I} \nabla_{\mathbf{x}} f_{j}\left(\mathbf{x}_{i}^{t}, \xi^{t}\right)$, this can be done updating $\mathbf{y}_{i}^{t}$ according to:

$$
\mathbf{y}_{i}^{t} \triangleq \sum_{j=1}^{I} w_{i j} \mathbf{y}_{j}^{t-1}+\nabla f_{i}\left(\mathbf{x}_{i}^{t}, \xi^{t}\right)-\nabla f_{i}\left(\mathbf{x}_{i}^{t-1}, \xi^{t-1}\right)
$$

with $\mathbf{y}_{i}^{0} \triangleq \nabla f_{i}\left(\mathbf{x}_{i}^{0}, \xi^{0}\right)$. Thus, given $\mathbf{y}_{i}^{t}$ in (8), the local estimates at node $i$ for $\pi_{i}\left(\mathbf{x}_{i}^{t}, \xi^{t}\right)$ in (7) and for $\nabla F\left(\mathbf{x}_{i}^{t}, \xi^{t}\right)$ in (4) are given by:

$$
\tilde{\pi}_{i}^{t}=I \mathbf{y}_{i}^{t}-\nabla, f_{i}\left(\mathbf{x}_{i}^{t}, \xi^{t}\right), \quad \widetilde{\nabla F}\left(\mathbf{x}_{i}^{t}, \xi^{t}\right)=I \mathbf{y}_{i}^{t},
$$

respectively. Note that since the weights $w_{i j}$ are constrained by the network topology, the update of $\mathbf{y}_{i}^{t}$ in (8), and thus $\tilde{\pi}_{i}^{t}$ and $\widetilde{\nabla F}\left(\mathbf{x}_{i}^{t}, \xi^{t}\right)$ in (9), can be now performed locally with message exchanges with the agents in the neighborhood $\mathcal{N}_{i}$.

Step 4 (Agreement): To force the asymptotic agreement among the $\mathbf{x}_{i}$ 's, a consensusbased step is employed on $\widehat{\mathbf{x}}_{i}^{t}$,s. Each agent $i$ updates its $\mathbf{x}_{i}$ as:

$$
\mathbf{x}_{i}^{t+1}=\sum_{j=1}^{I} w_{i j} \widehat{\mathbf{x}}_{j}\left(\mathbf{x}_{j}^{t}\right)
$$

which can be implemented via local message exchanges in each node's neighborhood. 


\subsection{The S-NEXT algorithm}

We are now in the position to formally introduce the S-NEXT algorithm, Algorithm 1; its convergence to stationary solutions of Problem (1) is stated in Theorem 2, whose proof is omitted because of space limitations. S-NEXT algorithm builds on the iterates (6) (wherein $\pi_{i}\left(\mathbf{x}_{i}^{t}, \xi^{t}\right)$ is replaced by $\tilde{\pi}_{i}^{t}$ in (9)), (4) (wherein $\nabla F\left(\mathbf{x}_{i}^{t}, \xi^{t}\right)$ is replaced by $\widetilde{\nabla F}\left(\mathbf{x}_{i}^{t}, \xi^{t}\right)$ in (9)), (8) and (10) introduced in the previous section. Also, in S1, in addition to solving the strongly convex optimization problem (6), we also introduced a step-size sequence $\alpha^{t}$ in the iterate: the new point $\mathbf{z}_{i}^{t}$ is a convex combination of the current estimate $\mathbf{x}_{i}^{t}$ and the solutions of (6). The convergence properties of S-NEXT are illustrated in the following Theorem.

Theorem 2 Given Problem (1) under A1-A7, let $\left\{\mathbf{x}^{t}\right\}_{n} \triangleq\left\{\left(\mathbf{x}_{i}^{t}\right)_{i=1}^{I}\right\}_{n}$ be the sequence generated by Algorithm 1, and let $\left\{\overline{\mathbf{x}}^{t}\right\}_{t} \triangleq\left\{(1 / I) \sum_{i=1}^{I} \mathbf{x}_{i}^{t}\right\}_{t}$ be its average. Choose the step-size sequences $\left\{\alpha^{t}\right\}_{t}$ and $\left\{\rho^{t}\right\}_{t}$ so that:

- $\alpha^{t} \in(0,1] \forall t, \sum_{t=0}^{\infty} \alpha^{t}=\infty$, and $\sum_{t=0}^{\infty}\left(\alpha^{t}\right)^{2}<\infty$;

- $\rho^{t} \in(0,1] \forall t, \quad \sum_{t=0}^{\infty} \rho^{t}=\infty$, and $\sum_{t=0}^{\infty}\left(\rho^{t}\right)^{2}<\infty$;

- $\lim _{t \rightarrow \infty} \alpha^{t} / \rho^{t}=0$

Then, we have:

(a) [convergence]: the sequence $\left\{\overline{\mathbf{x}}^{t}\right\}_{t}$ is bounded and all its limit points are stationary solutions of (1) almost surely;

(b) [consensus]: all the sequences $\left\{\mathbf{x}_{i}^{t}\right\}_{t}$ asymptotically agree, i.e., $\left\|\mathbf{x}_{i}^{t}-\overline{\mathbf{x}}^{t}\right\| \underset{t \rightarrow \infty}{\longrightarrow} 0$, for all $i=1, \ldots, I$.

\section{Application to Distributed Stochastic Training of Neural Networks}

As a specific application of the S-NEXT framework, we consider the distributed training of neural network (NN) models, a problem of significant practical interest $[25,26]$. Let us then assume a scenario where $I$ agents collect input-output pairs $\left(y_{i, m}, \mathbf{x}_{i, m}\right)$, for $m \in \mathcal{S}_{i}$, $i=1, \ldots, N$. Also, let us denote by $g(\mathbf{w}, \mathbf{x})$ a generic neural network with weight vector parameter $\mathbf{w}$, and taking $\mathbf{x}$ as input. Then, the distributed training problem can be mathematically cast as [25]:

$$
\min _{\mathbf{w}} \sum_{i=1}^{I} \underbrace{\frac{1}{\left|\mathcal{S}_{i}\right|} \sum_{m \in \mathcal{S}_{i}} l\left(y_{i, m}, g\left(\mathbf{w}, \mathbf{x}_{i, m}\right)\right)}_{f_{i}(\mathbf{w})}+G(\mathbf{w}),
$$


Algorithm 1 : Stochastic In-Network Nonconvex Optimization

Data: $\alpha^{t}, \rho^{t}>0, \mathbf{x}_{i}^{0} \in \mathcal{K}, \mathbf{y}_{i}^{0}=\nabla f_{i}\left(\mathbf{x}_{i}^{0}, \xi^{0}\right), \tilde{\pi}_{i}^{0}=(I-1) \mathbf{y}_{i}^{0}, \mathbf{d}_{i}^{0}=I \cdot \mathbf{y}_{i}^{0}$, for $i \in \mathcal{V}$; Set $t=0$;

(S1) SCA Optimization: Each agent $i$ evaluates:

$$
\begin{gathered}
\widehat{\mathbf{x}}_{i}^{t}=\underset{\mathbf{x}_{i} \in \mathcal{K}}{\operatorname{argmin}}\left\{\rho^{t}\left(\widetilde{f}_{i}\left(\mathbf{x}_{i} ; \mathbf{x}_{i}^{t}, \xi^{t}\right)+\tilde{\pi}_{i}^{t T}\left(\mathbf{x}_{i}-\mathbf{x}_{i}^{t}\right)\right)+\left(1-\rho^{t}\right) \mathbf{d}_{i}^{t^{T}}\left(\mathbf{x}_{i}-\mathbf{x}_{i}^{t}\right)+G\left(\mathbf{x}_{i}\right)\right\} \\
\mathbf{z}_{i}^{t}=\mathbf{x}_{i}^{t}+\alpha^{t}\left(\widehat{\mathbf{x}}_{i}^{t}-\mathbf{x}_{i}^{t}\right)
\end{gathered}
$$

(S2) Agreement and Gradient Tracking: Each agent $i$ collects data from its neighbors and updates the variables $\mathbf{x}_{i}^{t}, \mathbf{y}_{i}^{t}$, and $\tilde{\pi}_{i}^{t}$ as:

$$
\begin{aligned}
& \mathbf{x}_{i}^{t+1}=\sum_{j \in \mathcal{N}_{i}^{t}} w_{i j}^{t} \mathbf{z}_{j}^{t} \\
& \mathbf{y}_{i}^{t+1}=\sum_{j \in \mathcal{N}_{i}^{t}} w_{i j}^{t} \mathbf{y}_{j}^{t}+\nabla f_{i}\left(\mathbf{x}_{i}^{t+1}, \xi^{t+1}\right)-\nabla f_{i}\left(\mathbf{x}_{i}^{t}, \xi^{t}\right) \\
& \tilde{\pi}_{i}^{t+1}=I \cdot \mathbf{y}_{i}^{t+1}-\nabla f_{i}\left(\mathbf{x}_{i}^{t+1}, \xi^{t+1}\right)
\end{aligned}
$$

(S3) Gradient Averaging: Each agent $i$ updates the local variable $\mathbf{d}_{i}^{t}$ as:

$$
\mathbf{d}_{i}^{t+1}=\left(1-\rho^{t}\right) \mathbf{d}_{i}^{t}+\rho^{t} I \cdot \mathbf{y}_{i}^{t+1}
$$

(S4) If $\left(\mathbf{x}_{i}[n]\right)_{i}$ satisfies a termination rule, STOP; otherwise, $t \leftarrow t+1$ and go to (S.1).

where $l$ is a convex loss function (e.g., squared loss, cross-entropy, etc.), and $G$ is a convex regularizer (e.g., the $\ell_{2}$ norm). When the size of the dataset becomes very large, direct optimization of (11) might become prohibitive. A common approach to reduce complexity is to draw random mini-batch of data at every iteration, say, $\mathcal{B}_{i}^{t} \subseteq \mathcal{S}_{i}$, in order to approximate the global cost in (11). This approach leads to the following stochastic optimization problem:

$$
\min _{\mathbf{w}} \mathbb{E}[\sum_{i=1}^{I} \underbrace{\frac{1}{\left|\mathcal{B}_{i}^{t}\right|} \sum_{m \in \mathcal{B}_{i}^{t}} l\left(y_{i, m}, g\left(\mathbf{w}, \mathbf{x}_{i, m}\right)\right)}_{f_{i}(\mathbf{w}, \xi)}]+G(\mathbf{w}),
$$

which we aim to solve in a distributed fashion using the S-NEXT framework. Indeed, each function $f_{i}(\mathbf{w}, \xi)$ in (12) is nonconvex beecause of the presence of the NN function $g$, and is dependent on a random parameter $\xi$ that models the random data sampling at each iteration. Our proposed approach is to use the S-NEXT algorithm to solve (12), using

$$
\tilde{f}_{i}\left(\mathbf{w}_{i} ; \mathbf{w}_{i}^{t}, \xi^{t}\right)=\frac{1}{\left|\mathcal{B}_{i}^{t}\right|} \sum_{m \in \mathcal{B}_{i}^{t}} l\left(y_{i, m}, \widetilde{g}\left(\mathbf{w}_{i} ; \mathbf{w}_{i}^{t}, \mathbf{x}_{i, m}\right)\right)+\frac{\tau}{2}\left\|\mathbf{w}-\mathbf{w}_{i}^{t}\right\|^{2}
$$

as a local strongly convex surrogate function for $f_{i}(\mathbf{w}, \xi)$, where $\tau>0$, and

$$
\widetilde{g}\left(\mathbf{w} ; \mathbf{w}_{i}^{t}, \mathbf{x}_{i, m}\right)=g\left(\mathbf{w}_{i}^{t}, \mathbf{x}_{i, m}\right)+\nabla g\left(\mathbf{w}_{i}^{t}, \mathbf{x}_{i, m}\right)^{T}\left(\mathbf{w}_{i}-\mathbf{w}_{i}^{t}\right)
$$


represents the linearization of the NN function $g$ around $\mathbf{w}_{i}^{t}$, for a given input $\mathbf{x}_{i, m}$. The terms $\nabla g\left(\mathbf{w}_{i}^{t}, \mathbf{x}_{i, m}\right)$, for all $i$ and $m$, can be computed by standard back-propagation [27].

A practical example. Consider $l(a, b)=(a-b)^{2}$ and $G(\mathbf{w})=\lambda\|\mathbf{w}\|^{2}$ in (12). This setting is largely used in regression type problems. Now, letting $\mathbf{J}_{i, m}^{t}=\nabla g\left(\mathbf{w}_{i}^{t}, \mathbf{x}_{i, m}\right)$ and $r_{i, m}^{t}=$ $y_{i, m}-g\left(\mathbf{w}_{i}^{t} ; \mathbf{x}_{i, m}\right)+\mathbf{J}_{i, m}^{t}{ }^{T} \mathbf{w}_{i}^{t}$, we have the closed-form solution for the SCA optimization in Algorithm 1:

$$
\widehat{\mathbf{w}}_{i}^{t}=\left(\mathbf{A}_{i}^{t}\right)^{-1} \mathbf{b}_{i}^{t}
$$

where $\mathbf{A}_{i}^{t}$ and $\mathbf{b}_{i}^{t}$ write as:

$$
\begin{aligned}
& \mathbf{A}_{i}^{t}=\frac{\rho^{t}}{\left|\mathcal{B}_{i}^{t}\right|} \sum_{m \in \mathcal{B}_{i}^{t}} \mathbf{J}_{i, m}^{t} \mathbf{J}_{i, m}^{t}{ }^{T}+\lambda \mathbf{I}, \\
& \mathbf{b}_{i}^{t}=\frac{\rho^{t}}{\left|\mathcal{B}_{i}^{t}\right|} \sum_{m \in \mathcal{B}_{i}^{t}} \mathbf{J}_{i, m}^{t} r_{i, m}^{t}-\frac{\rho^{t}}{2} \tilde{\pi}_{i}^{t}-\frac{\left(1-\rho^{t}\right)}{2} \mathbf{d}_{i}(t) .
\end{aligned}
$$

The step in (14) is in closed-form, but requires the inversion of a matrix at each iteration. The complexity is of the order of $O\left(p^{3}\right)$, where $p$ is the size of the vector $\mathbf{w}$. As shown in $[13,25]$, this complexity can be largely reduced exploiting either inexact updates or parallel computation using multiple cores at each network agent.

\section{Numerical Results}

In this section, we evaluate numerically the performance of the S-NEXT algorithm, considering the practical case elaborated in the previous Section (i.e., the update in (14)), and applying it to two regression problems: the Boston dataset ${ }^{1}$, and the SML2010 dataset ${ }^{2}$. In both cases, we randomly distribute the training examples on a connected network composed of 6 agents and having random topology. Regarding the neural network architecture, we consider models with two hidden layers having 30 units each, and tanh non-linearities. In Fig. 1, we compare the learning rate of S-NEXT with a distributed stochastic gradient descent (SGD) procedure [16]. We also consider three additional strong baselines, which are centralized implementations of SGD, SCA [23], and Adam [28]. In these centralized cases, we assume all training data available at a single processing unit that performs standard optimization with these baselines. The regularization parameter is set to $\lambda=10^{-2}$. The parameter setting for S-NEXT considers a time varying step-size rule given by $\alpha^{t}=\alpha^{t-1}\left(1-\varepsilon \alpha^{t-1}\right)$, having initial learning rate of $\alpha^{0}=0.01$, and decaying factor $\varepsilon=10^{-3}$; similarly, $\rho^{t}$ follows the same decaying rule with $\rho^{0}=0.9$ and $\varepsilon=5 \times 10^{-4}$. The learning rates and the hyperparameters of all other methods are fine-tuned to provide the fastest convergence behavior. We implement the simulation in the JAX framework [29]. ${ }^{3}$ As we can notice from Fig. 1, the proposed method converges to the solution of its centralized counterpart, i.e., the stochastic

\footnotetext{
${ }^{1}$ https://www.cs.toronto.edu/ delve/data/boston/bostonDetail.html

${ }^{2}$ https://archive.ics.uci.edu/ml/datasets/SML2010

${ }^{3}$ https://jax.readthedocs.io/
} 


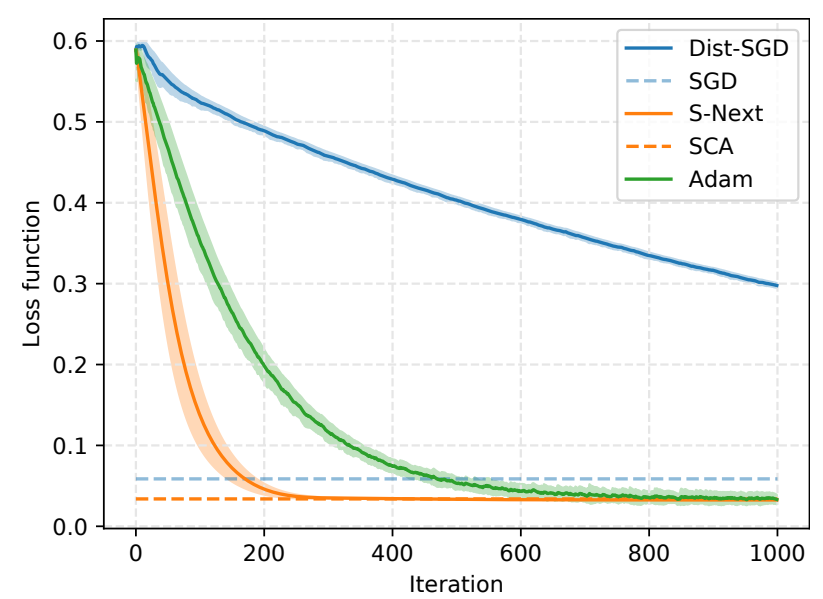

(a) Boston

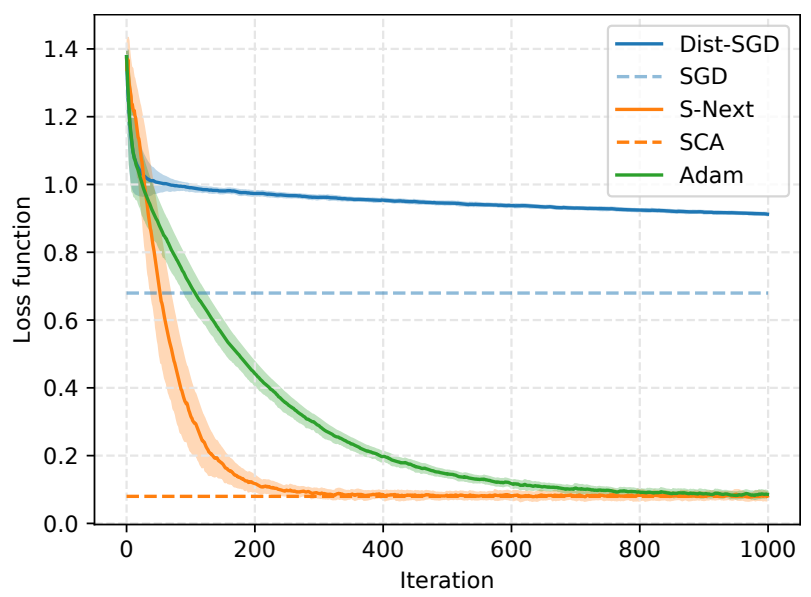

(b) SML2010

Figure 1: Learning curve of different algorithms applied to prediction tasks

SCA method [23], which is provably convergent to stationary solutions of problem (12). This confirms the theoretical results of Theorem 2. Furthermore, when compared to distributed SGD, our method illustrates a much faster convergence behavior to generally better locally optimal solutions of (12). Interestingly, S-NEXT outperforms also the centralized Adam algorithm from [28] in terms of learning rate. These results illustrate the very good performance of the proposed S-NEXT algorithm, when applied to the distributed stochastic training of NN models.

\section{Conclusions}

In this paper we have introduced S-NEXT, a novel algorithmic framework for stochastic nonconvex distributed optimization in multi-agent networks. S-NEXT exploits successive convex approximation techniques while leveraging dynamic consensus as a gradient tracking mechanism, and recursive average to asymptotically evaluate the expected gradient of the network loss function. Almost sure convergence to (stationary) solutions of the nonconvex problem is established under mild conditions. The proposed method is then customized to the stochastic training of NN models over a multi-agent network. Numerical results confirm the theoretical findings, and show that S-NEXT compares favorably to existing algorithms for distributed nonconvex stochastic optimization and learning.

\section{References}

[1] A. Nedić and A. Ozdaglar, "Distributed subgradient methods for multiagent optimization," IEEE Trans. on Automatic Control, vol. 54, no. 1, pp. 48-61, Jan. 2009. 
[2] A. Nedić, A. Ozdaglar, and P. Parillo, "Constrained consensus and optimization in multiagent networks," IEEE Trans. on Automatic Control, vol. 55, no. 4, pp. 922-938, 2010.

[3] D. Jakovetić, J. Xavier, and J. M. Moura, "Fast distributed gradient methods," IEEE Transactions on Automatic Control, vol. 59, no. 5, pp. 1131-1146, 2014.

[4] S. Boyd, N. Parikh, E. Chu, B. Peleato, and J. Eckstein, Distributed optimization and statistical learning via the alternating direction method of multipliers, ser. Foundations and Trends in Machine Learning. Boston-Delft: NOW Publishers, 2011, vol. 3, no. 1.

[5] F. S. Cattivelli and A. H. Sayed, "Diffusion LMS strategies for distributed estimation," IEEE Trans. on Signal Processing, vol. 58, pp. 1035-1048, March 2010.

[6] J. Chen and A. H. Sayed, "Diffusion adaptation strategies for distributed optimization and learning over networks," IEEE Trans. on Signal Processing, vol. 60, no. 8, pp. 42894305, August 2012.

[7] P. Di Lorenzo and A. H. Sayed, "Sparse distributed learning based on diffusion adaptation," IEEE Trans. on Signal Processing, vol. 61, no. 6, pp. 1419-1433, March 2013.

[8] S. Vlaski, L. Vandenberghe, and A. H. Sayed, "Diffusion stochastic optimization with non-smooth regularizers," in 2016 IEEE International Conference on Acoustics, Speech and Signal Processing (ICASSP). IEEE, 2016, pp. 4149-4153.

[9] H. Ouyang, N. He, L. Tran, and A. Gray, "Stochastic alternating direction method of multipliers," in International Conference on Machine Learning, 2013, pp. 80-88.

[10] W. Zhong and J. Kwok, "Fast stochastic alternating direction method of multipliers," in International Conference on Machine Learning, 2014, pp. 46-54.

[11] J. Zeng and W. Yin, "On nonconvex decentralized gradient descent," IEEE Trans. on Signal Processing, vol. 66, no. 11, pp. 2834-2848, 2018.

[12] H.-T. Wai, J. Lafond, A. Scaglione, and E. Moulines, "Decentralized Frank-Wolfe algorithm for convex and nonconvex problems," IEEE Trans. on Automatic Control, vol. 62, no. 11, pp. 5522-5537, 2017.

[13] P. Di Lorenzo and G. Scutari, "Next: In-network nonconvex optimization," IEEE Transactions on Signal and Information Processing over Networks, vol. 2, no. 2, pp. 120-136, 2016.

[14] M. Hong, D. Hajinezhad, and M.-M. Zhao, "Prox-PDA: The proximal primal-dual algorithm for fast distributed nonconvex optimization and learning over networks," in Proc. of ICML, 2017, pp. 1529-1538.

[15] B. Swenson, S. Kar, H. V. Poor, and J. Moura, "Annealing for distributed global optimization," arXiv preprint arXiv:1903.07258, 2019. 
[16] S. Vlaski and A. H. Sayed, "Distributed learning in non-convex environments-part i: Agreement at a linear rate," arXiv preprint arXiv:1907.01848, 2019.

[17] — - "Distributed learning in non-convex environments-part ii: Polynomial escape from saddle-points," arXiv preprint arXiv:1907.01849, 2019.

[18] — - "Second-order guarantees of stochastic gradient descent in non-convex optimization," arXiv preprint arXiv:1908.07023, 2019.

[19] J. George, T. Yang, H. Bai, and P. Gurram, "Distributed stochastic gradient method for non-convex problems with applications in supervised learning," arXiv preprint arXiv:1908.06693, 2019.

[20] S. Lu, X. Zhang, H. Sun, and M. Hong, "Gnsd: a gradient-tracking based nonconvex stochastic algorithm for decentralized optimization," in Proceedings of IEEE Data Science Workshop (DSW), 2019, pp. 315-321.

[21] G. Scutari, F. Facchinei, P. Song, D. P. Palomar, and J.-S. Pang, "Decomposition by partial linearization: Parallel optimization of multiuser systems," IEEE Trans. on Signal Processing, vol. 63, no. 3, pp. 641-656, Feb. 2014.

[22] F. Facchinei, G. Scutari, and S. Sagratella, "Parallel selective algorithms for nonconvex big data optimization," IEEE Trans. on Signal Processing, vol. 63, no. 7, pp. 1874-1889, April 2015.

[23] Y. Yang, G. Scutari, D. P. Palomar, and M. Pesavento, "A parallel decomposition method for nonconvex stochastic multi-agent optimization problems," IEEE Transactions on Signal Processing, vol. 64, no. 11, pp. 2949-2964, 2016.

[24] M. Zhu and S. Martínez, "Discrete-time dynamic average consensus," Automatica, vol. 46, no. 2, pp. 322-329, Feb. 2010.

[25] S. Scardapane and P. Di Lorenzo, "A framework for parallel and distributed training of neural networks," Neural Networks, vol. 91, pp. 42-54, 2017.

[26] W. Wen, C. Xu, F. Yan, C. Wu, Y. Wang, Y. Chen, and H. Li, “Terngrad: Ternary gradients to reduce communication in distributed deep learning," in Advances in neural information processing systems, 2017, pp. 1509-1519.

[27] B. M. Wilamowski, N. J. Cotton, O. Kaynak, and G. Dundar, "Computing gradient vector and jacobian matrix in arbitrarily connected neural networks," IEEE Transactions on Industrial Electronics, vol. 55, no. 10, pp. 3784-3790, 2008.

[28] D. P. Kingma and J. Ba, "Adam: A method for stochastic optimization," arXiv preprint arXiv:1412.6980, 2014. 
[29] J. Bradbury, R. Frostig, P. Hawkins, M. J. Johnson, C. Leary, D. Maclaurin, and S. Wanderman-Milne, "JAX: composable transformations of Python+NumPy programs," 2018. 